\title{
Virtual Reality Exergame in older Patients with Hypertension: A Preliminary Study to determine Load Intensity and Blood Pressure
}

\section{Susan Vorwerg ( $\sim$ susan.vorwerg@charite.de)}

Charité - Universitätsmedizin Berlin, corporate member of Freie Universität Berlin and HumboldtUniversität zu Berlin

\section{Oskar Stamm}

Charité - Universitätsmedizin Berlin, corporate member of Freie Universität Berlin and HumboldtUniversität zu Berlin

\section{Michele Haink}

Charité - Universitätsmedizin Berlin, corporate member of Freie Universität Berlin and HumboldtUniversität zu Berlin

\section{Research Article}

Keywords: VR training, head mounted display, physical activity, post-exercise hypotension, heart rate

Posted Date: January 27th, 2022

DOI: https://doi.org/10.21203/rs.3.rs-1284291/v1

License: (c) (1) This work is licensed under a Creative Commons Attribution 4.0 International License. Read Full License 


\section{Abstract}

\section{Background}

Lifestyle changes and physical activity can make an important contribution to reducing the risk factor of high blood pressure (BP). Whether virtual reality (VR) exergames are also appropriate and make a positive contribution to the reduction of BP has not yet been sufficiently investigated. Therefore, the aim of the study was to gain knowledge of the load intensities to be achieved during a VR exergame and to examine the short-term effects on BP.

\section{Methods}

For the preliminary study, 22 participants with hypertension over the age of 65 years were analyzed. The study took place in a mobile laboratory truck. All participants visited on two occasions. During visit 1 , a VR strength endurance training (VR-SET) was conducted and on visit 2 a VR endurance training (VR-ET) was performed. Each VR training session lasted around $25 \mathrm{~min}$ and was of a moderate intensity. Heart rate (HR) was measured across the entire training session, as well as BP before and after VR training. The RPE and task load were determined after VR training. Included in the statistical analysis were the ShapiroWilk test, the paired t-test, the Wilcoxon test and the ANOVA for repeated measures.

Results

During the "main part" ( $p<.001)$, at the "end" ( $p=.002)$ and for the "maximum HR" $(p<.001)$, significant load differences between both training sessions could be determined. In addition, significantly more participants in the VR-SET achieved the moderate load intensity of at least $40 \%$ of HRR ( $p=.014)$. Regarding RPE, participants rated their subjectively perceived exertion significantly higher in the VR-SET than in the VR-ET ( $p=.028)$. Systolic BP decreased significantly in both training sessions when compared before training and 5 min after training $(p=.015 ; p=.003)$, as well as before training and 10 min after training $(p=.018 ; p<.001)$.

\section{Conclusions}

The individual moderate load intensity of $40 \%$ can be reached during the VR-SET. Nevertheless, a positive short-term effect of VR training on BP behavior (post-exercise hypotension) was observed after both VR training sessions. The first results indicate that a VR exergame can lead to health-promoting effects in hypertension.

Trial registration

The study was registered in the German Clinical Trials Register (DRKS-ID: DRKS00022881).

\section{Introduction}


With more than 10 million deaths per year, hypertension is one of the most common preventable causes of death worldwide and is one of the most important risk factors for cardiovascular diseases (CVD). $[1,2]$ Especially in the over 60 age-group, around two thirds of these individuals suffer from hypertension. $[2,3]$ Due to the high degree of associated morbidity and mortality, as well as the increasing prevalence in old age, hypertension is additionally a relevant cost factor.[4] For example, in the USA, hypertension leads to around 300,000 hospitalizations annually and is associated with approximately 53 billion dollars in direct costs.[3]

However, hypertension can be influenced by individual health behavior. Lifestyle changes, such as dietary changes, restrictions on alcohol and tobacco consumption and above all physical activity, can lead to antihypertensive effects and minimize cardiovascular risk factors.[4] In particular, the positive influence of physical activity on BP has been demonstrated by various epidemiological studies.[5-7] A metaanalysis of RCTs has shown that aerobic endurance training, dynamic resistance training and isometric training reduce resting systolic BP/diastolic BP by $3.5 / 2.5,1.8 / 3.2$ and by $10.9 / 6.2 \mathrm{mmHg}$, respectively. [6] According to these studies, a combination of aerobic exercise and resistance training in older individuals provides the best effect in lowering $\mathrm{BP}$, increasing arterial elasticity and thus reducing the risk of CVD.[5] In this regard, guidelines from the American College of Cardiology (ACC)/American Heart Association (AHA), International Society of Hypertension (ISH) or the European Society of Cardiology (ESC)/European Society of Hypertension (ESH) recommend that individuals with hypertension should perform at least 30 min of dynamic aerobic exercise (walking, jogging, cycling or swimming) at a moderate intensity five to seven days per week, as well as resistance exercise two to three days per week. [8-10] Although older adults' physical activity has increased significantly since 1998 in Germany, the recommendations are not being regularly achieved.[11] Around $70 \%$ of people over the age of 65 are physically active for less than 2.5 hours per week.[11] In this context, exergames could make an important contribution to motivating older adults to become more physically active. The increased motivation aspect has been confirmed in studies and has also resulted in an increase in well-being and enjoyment.[12-14] However, there is limited research on exergaming and hypertension in older adults. Studies involving young adults/students which examined BP during exergaming, identified positive effects on BP.[15-17] Hypertension was not previously considered a defined inclusion criterion and could not be found in any previous studies with exergames.[18] Therefore, one of the aims of the study is to gather initial insights into the influence of a VR exergame on the BP of older patients with hypertension. Furthermore, it is not known from previous studies whether a VR exergame is capable of achieving the required moderate load intensity in older adults with hypertension. In this context, a study by Silva et al., involving adult students, indicated that on average, lower HR values of below $90 \mathrm{bpm}$ were achieved during VR exergaming.[17] However, maintaining the intensity for optimal training and thus avoiding over and underload was crucial to achieving positive health effects among older patients with hypertension. [19]

Consequently, the aim of the study was to investigate the load intensity of older patients with hypertension during training in a VR exergame. To achieve this goal, two training concepts in VR, 1. VR strength endurance training (VR-SET) and 2. VR endurance training (VR-ET) were tested and compared. 
Thus, the following primary research question was to be answered: is the load intensity of the VR exergame appropriate, in order that older patients with hypertension can exercise within their individually calculated moderate load intensity of $40 \%$ to $60 \%$ during VR training? The following main hypotheses were derived from this:

$\mathrm{HO}_{1 \mathrm{a}}$ : During the "main part" of the VR, the individual moderate load intensity of at least $40 \%$ could not be significantly achieved in the VR-SET or the VR-ET.

$\mathrm{HO}_{1 \mathrm{~b}}$ : There is no significant difference between the VR-SET and the VR-ET in terms of achieving moderate individual load intensities during the "main part".

$\mathrm{HO}_{1 \mathrm{c}}$ : There are no significant differences in $\mathrm{HR}$ values for the various training sections ("start", "warmup", "main part", "cool down", "end") during the VR-ET and VR-SET programs, based on gender, VR experience, training frequency and age.

In addition, another aim of the study was to determine whether the participants assessed themselves adequately in relation to their objectively measured exertion via HR. The following hypotheses should be answered.

$\mathrm{HO}_{2 \mathrm{a}}$ : There were no significant differences between the objectively measured $\mathrm{HR}$ and subjectively RPE during the "main part" of the VR-SET and the VR-ET.

$\mathrm{HO}_{2 \mathrm{~b}}$ : The perceived exertion did not differ significantly between the VR-SET and the VR-ET.

As already mentioned in the introduction, BP will be investigated in addition to load intensity. In particular, the question arises whether the effects of post-exercise hypotension can be detected. Therefore, the following hypotheses were defined.

$\mathrm{HO}_{3 \mathrm{a}}$ : No significant changes in $\mathrm{BP}$ values (systolic, diastolic, pulse) could be detected either before or after training in the case of the VR-SET or the VR-ET.

$\mathrm{HO}_{3 \mathrm{~b}}$ : The BP values did not differ significantly between the VR-SET and the VR-ET at the individual time points.

A final research question was related to the perceived task load. How do older individuals rate the mental, physical and time demands as well as the effort of the VR exergame?

$\mathrm{HO}_{4}$ : No significant differences in perceived task load (mental, physical, temporal demand and effort) were found between the VR-SET and the VR-ET.

\section{Methods}


The study was a preliminary study evaluating a VR exergame involving participants over the age of 65 years, who had been diagnosed with essential hypertension. Data were collected prospectively during two visits.

\section{General}

All participants were recruited through the internal volunteer database of the Geriatrics Research Group of the Charité Berlin, certain sports centers and geriatric organizations. There were no personal relationships between the study team and the participants. To recruit potential participants, telephone calls were made and e-mails with study information were sent $(n=174)$. For the study, 32 potential participants were recruited and checked for inclusion and exclusion criteria by telephone (Table 1).

\section{Table 1. Inclusion and exclusion criteria of the study.}

\begin{tabular}{|ll|}
\hline inclusion criteria & exclusion criteria \\
\hline - $\geq 65$ years & - Presence of legal guardianship \\
$\begin{array}{l}\text { hypertension (stage I) } \\
\text { - Independent mobility }\end{array}$ & - MCl and severe cognitive impairment (TICS < 33 points). \\
- Presence of a signed informed & - Severe visual impairment \\
consent form & - Travel sickness \\
- One training session was & - Medical conditions associated with high risk of falls (stroke, \\
completely finished & Parkinson's disease) \\
& - Increased risk of falling (Tinetti test < 24 points) \\
\hline
\end{tabular}

For this purpose, among other things, the TICS-Telephone Interview for Cognitive Status[20] was used. However, due to the worsening pandemic situation, seven of the participants recruited withdrew from the study. A total of 24 participants took part. Two participants dropped out of the study due to VR sickness symptoms at the beginning of the first training session (Fig. 1).

\section{Fig. 1. Flow diagram of the recruitment process, visits and assessments.}

The study took place in a mobile research laboratory VITALab.Mobile[21] in the form of a truck on the campus of the Evangelisches Geriatriezentrum in Berlin (Fig. 2). During the study period from 10-05-20 to 11-06-20, the participants made two individual visits within two weeks. Each participant tested the VRSET on week one (visit 1) and the VR-ET on week two (visit 2). The study was organized, supervised and conducted by two research assistants with a background in physical therapy and one trained public health master's student. The study design did not include randomization and blinding of participants and study personnel. After all included participants had completed visit 2, the study terminated. 
Fig. 2. Mobile research laboratory VITALab.mobile.

\section{Procedure}

On the first day of the study, all participants were informed in detail about the study procedure, after which they gave their written consent to participate in the study. In addition, the Tinetti test[22] was performed to eliminate the risk of falls. The participants also answered a questionnaire to collect framework data and a BP measurement was taken three times before the VR training session. Therefore, the BM28 HSD upper arm BP monitor from Beurer GmbH was used on the left upper arm. For both VR training sessions, a Polar $\mathrm{M} 600^{\mathrm{TM}}$ smartwatch was placed on the participants' wrists to monitor their HR over the entire training session. Subsequently, the HTC Vive Pro ${ }^{T M}$ was explained to the participants, the trackers were attached to their hands and the VR headset was put on.

The VR training session lasted between 20 and 25 min and was divided into five sections: 1. introduction by the trainer agent named "Anna", 2. warm up, 3. main part, 4. cool down, 5. training analysis. Only section 3, "main part", differed between the two training sessions. The "main part" focused on moderate intensity (40-60\% of heart rate reserve (HRR)) according to the recommendations of the American College of Sport Medicine (ACSM) for patients with hypertension.[23] The other four sections remained identical across both visits (Fig. 3).

\section{Fig. 3. Section 1, 2, 4 and 5 of the VR Exergame.}

The "main part" of the VR-SET was divided into a learning phase and an exercise phase and lasted approximately 15 to $20 \mathrm{~min}$. For the VR-SET, a chair as well as dumbbells were used as training objects. In the learning phase the participants performed the following five exercises (Fig. 4). After an initial demonstration by Anna, the participants were invited to repeat the exercise three times. During the exercise phase, the five exercises were executed in two sets. Each exercise was repeated 12 to 20 times. Between the exercises was a short break of $20 \mathrm{sec}$. An active break of 1 min took place after the first set. Within this time, the participants had to make as many colored balls as possible vanish by touching the balls with their hands.

\section{Fig. 4. Section 3 - training phase of the VR-SET (visit 1).}

During the "main part" of the VR-ET, three exercises were completed (Fig. 5). For this training session Valve Index ${ }^{\mathrm{TM}}$ controllers were used. In the "ball game", a ball was grasped with either the left or the right hand and thrown into a ring. After three hits, Anna changed the position of the ring. During the "high-five" exercise, Anna's movements had to be mirrored, which involved touching two pink balls with both hands at the same time. When this was mastered successfully, Anna changed to another position. During the "dancing" session, the VR environment was transformed into a 70s disco. The participants learned a specific sequence of steps consisting of four elements (basic step, cross step, travolta and mixer) and practiced these in time with the music. 
Fig. 5. Section 3 - training phase of the VR-ET (visit 2).

After both VR training sessions, BP was measured immediately after the training session and after five as well as $10 \mathrm{~min}$. This was done three times in total at each time point. In addition, the Borg's RPE scale[24] and NASA TXL (Task Load Index)[25] were performed, and questions relating to the total duration and break times were answered. After each training session, further assessments were performed, which form part of other publications.[26-28]

\section{Materials}

An HTC Vive Pro ${ }^{T M}$ VR headset with a field of view of 110 degrees was used for the study. In addition, two Valve Index Base Stations 2.0 were positioned diagonally to one other under the ceiling of the truck. To enable interactivity in the VR-SET, five HTC Vive Trackers $2.0^{\mathrm{TM}}$ were attached to the users' hands and to the training objects (dumbbells and chair). For the VR-ET, two Valve Index ${ }^{\mathrm{TM}}$ controllers were used to facilitate finger tracking and thus the gripping, releasing and throwing of the balls.

The Polar $\mathrm{M} 600^{\mathrm{TM}}$ smartwatch was used as a monitoring tool to record the participant's HR during the entire VR training. The HR was measured via an optical monitor with six LED sensors on the wrist via photoplethysmography.[29] Polar M600 has a sampling rate of $60 \mathrm{~Hz}$. The HR was displayed to the participants in the VR environment via an interface to the VR application. Afterwards, the collected data were transferred to the Polar Flow app via Bluetooth and downloaded as a CSV file. Existing studies show that the Polar M600 can be used as a valid measurement tool.[30,31]

\section{Statistical methods}

Data preparation The determined HR data were prepared in Excel and divided into the corresponding training sections ("start", "warm-up", "main part", "cool down" and "end"). The basis for the determination of the load intensity of the participants was the formula of Sally Edwards to calculate the maximum HR (men: HRmax $=214-0.5 \times$ age $-0.11 \times$ bodyweight in kg, women: HRmax $=210-0.5 \times$ age $-0.11 \times$ bodyweight in $\mathrm{kg}$ ).[32] Subsequently, the difference between the maximum HR and the resting HR (HRR) was used to determine the individual moderate load intensity from 40 to $60 \%$ for each participant, using the Karvonen formula (HRtraining $=(($ HRmax-HRresting $) *$ intensity\% $)+$ HRresting $).$ [33] In addition, the values of Borg's RPE scale (value range from 6 to 20 points) was converted into HR values (60 to 200 bpm) for comparison with the objectively measured HR via the Polar M600.

Data analysis was performed by descriptive and inductive statistical methods, using IBM SPSS Statistics 27. Mean values, standard deviations and minimum and maximum values were presented descriptively. For the purposes of inductive statistics, the data were examined for normal distribution using the ShapiroWilk test. To test for mean differences, the paired t-test was used under normally distributed conditions. Should there be no normal distribution or the data were interval scaled, the Wilcoxon test was used. 
Repeated measures were tested with ANOVA. A normal distribution of the standardized residuals was given. If sphericity was not assumed, the Greenhouse-Geisser correction was applied. Furthermore, between-subject effects were examined when comparing groups. Confidence intervals $(\mathrm{Cl} 95 \%)$ and effect sizes $(d, r)$ were also provided according to the interpretation of Cohen (1988)[34]. No groups were formed beforehand. All 22 subjects were treated identically during the two visits. Due to connection problems between the Polar M600 and the HTC vive system, there was a missing value for the HR. This was countered with a list-wise case exclusion. Therefore, certain results show an $n=21$. In the statistical analysis of the HR values, classifications were made according to frequency of activity, gender, subjects with and without VR experience and age-groups up to 76 and over 76 years of age. A sample size calculation a priori, with a statistical power of 0.80 , an effect size of 0.5 and an alpha of $5 \%$ (differences between two dependent means, two tails) was performed and resulted in a total sample size of 34 . However, this sample size could not be met due to the refusal of certain potential participants to take part in the study as a result of the Covid-19 pandemic.

\section{Results}

\section{Participants}

Table 2. Characteristics of the sample ( $n=22)$.

\begin{tabular}{|c|c|c|c|c|}
\hline & $\min$ & $\max$ & $M$ & SD \\
\hline Age & 65.00 & 80.00 & 74.50 & 3.64 \\
\hline Height, m & 1.54 & 1.85 & 1.71 & .10 \\
\hline Weight, kg & 60.00 & 103.00 & 76.18 & 12.54 \\
\hline $\mathrm{BMI}, \mathrm{kg} / \mathrm{m}^{2}$ & 20.75 & 32.51 & 26.10 & 3.52 \\
\hline HRresting, bpm & 51.00 & 85.00 & 63.55 & 7.62 \\
\hline HRmax, bpm & 161.87 & 173.69 & 166.01 & 2.54 \\
\hline $\mathrm{HR} 40 \%$, bpm & 95.35 & 117.47 & 104.53 & 4.86 \\
\hline HR60 \%, bpm & 117.52 & 133.70 & 125.02 & 3.64 \\
\hline
\end{tabular}

The results of 22 participants with an age of $74.50 \pm 3.64$ years were evaluated (Table 2). Nine participants were male and 13 were female. All participants had essential hypertension as per ICD-10-GM2021 110. Most of them were taking at least one of the following drugs: angiotensin II receptor blockers $(n=9)$, anticoagulants $(n=7)$, calcium channel blockers $(n=5)$, ACE inhibitors $(n=3)$, diuretics $(n=2)$, angiotensin-converting enzyme inhibitors $(n=1)$ or $\beta$-Acetyldigoxin $(n=1)$. Five participants were taking $\beta$ - 
Blockers. Three participants were not using any medication for the treatment of hypertension. One person reported occasional dizziness in daily life. Ten of the participants had previous VR experience, gained professionally (virtual built house) through other research projects or in museums and exhibitions.

Sixteen participants reported being active in sports more than once a week. Six participants were active in sports less than once a week. On average, the participants participated in sports 2.5 times per week (including strength training, dance classes, Pilates, cycling, rehabilitation sports, water gymnastics, meditation, Nordic walking, jogging, swimming, horseback riding and hiking). None of the participants had cognitive impairments, measured by the TICS ( $37.27 \pm 2.57$ points) or an increased risk of falling, measured by the Tinetti test $(27.64 \pm 0.79$ points).

\section{Load intensity}

The evaluation of the load intensity was based on the continuously recorded HR during the VR exergame. For this purpose, the mean values of the HR values of the different training sections were compared with each other (Table 3). Significant differences between the two training sessions were found in the mean value of the "main part" as well as at the "end". In addition, the maximum HR achieved during training differed significantly between the VR-SET and the VR-ET.

Table 3. Mean HR values of the VR-SET and VR-ET.

\begin{tabular}{|c|c|c|c|c|c|}
\hline & $\begin{array}{l}\text { VR-SET } \\
M(S D), b p m \\
n=21\end{array}$ & $\begin{array}{l}\text { VR-ET } \\
M(S D), b p m \\
n=21\end{array}$ & p-value & $95 \% \mathrm{Cl}$ & d \\
\hline Start & $86.14(14.12)$ & 83.05 (14.27) & .117 & $-.84,7.03$ & .358 \\
\hline Warm-up & 94.01 (16.99) & $93.04(14.02)$ & .686 & $-3.93,5.86$ & .090 \\
\hline Training phase & 106.08 (19.70) & 96.98 (15.16) & $.000 \star \star \star$ & $4.84,13.36$ & .973 \\
\hline Cool down & $104.42(20.25)$ & $103.51(15.64)$ & .687 & $-3.76,5.57$ & .089 \\
\hline End & 100.67 (19.73) & 93.33 (15.05) & $.002^{\star \star}$ & $3.14,11.52$ & .797 \\
\hline HRmax & $124.90(20.65)$ & $114.48(14.34)$ & $.000 \star \star \star$ & $5.93,14.93$ & 1.055 \\
\hline
\end{tabular}

As the mean values of the "main part" showed, higher loads were achieved in the VR-SET than in the VRET. In the "main part" of the VR-SET, half of the participants reached their personal intensity range of at least $40 \%$ (lower limit of the moderate target zone). In contrast, in the VR-ET, only five participants (27.73\%) managed to reach a load intensity of at least $40 \%$ (Fig. 6). 
Fig. 6. Progression of mean HR during training phase in VR-SET and VR-ET per participant. The black circles indicate exceeding an individual load intensity of $40 \%$. Participant 8 in Fig. B is a missing value.

According to the assumption of the primary hypothesis, $\mathrm{HO}_{1 \mathrm{a}}$, no significant achievement of the load intensity of at least $40 \%(M=104.53 \pm 4.56)$ could be determined for both VR training programs.

Regarding the VR-SET, there were no differences to the load limit of $40 \%$ in the "main part" $(n=22, M=$ $106.06 \pm 19.22$, paired t-test $p=.688,95 \% \mathrm{Cl}[-9.15,6.16], d=-.087)$ and even significantly lower load values were achieved in the "main part" of the VR-ET $(n=21, M=96.98 \pm 15.16$, paired t-test $p=.017$, $95 \% \mathrm{Cl}[1.53,13.68], \mathrm{d}=.570)$. Consequently, the $\mathrm{HO}_{1 \mathrm{a}}$ must be accepted. However, a comparison between the VR-SET and the VR-ET showed that significantly more participants reached the desired exercise intensity of $40-60 \%$ in the "main part" of the VR-SET than in the VR-ET $(n=21$, Wilcoxon test $p=0.014, r=$ -.534). Thus, hypothesis $\mathrm{HO}_{1 \mathrm{~b}}$ can be rejected.

In addition, various group comparisons were made to assess the load intensity during the different training sections. Within the group "training frequency" it was descriptively determined for both the VRSET and the VR-ET that participants who train less frequently (0-1x per week) have higher HR values than participants who train more frequently ( $2 x$ and more per week). Higher HR values were also determined within both training programs for the group of participants under 76 years of age and women, as well as for the group that had no previous VR experience. ANOVA with repeated measures found no statistically significant main effects between the groups for both training programs (Fig. 7). Hypothesis $\mathrm{HO}_{1 \mathrm{c}}$ must be accepted.

Fig. 7. Main Effects of ANOVA with repeated measures for groups in VR-SET and VR-ET.

\section{Perceived exertion}

The subjective perceived exertion was determined after each VR training session using Borg's RPE scale. The RPE ranged from "easy" to "somewhat difficult" in the VR-SET ( $M=12.14 \pm 1.81)$ and "easy" in the VRET $(M=10.82 \pm 2.69)$.

When comparing the converted RPE values with the actual measured HR values during the "main part", the values of RPE were higher than those of the objective measurement in both visits (Table 4). Contrary to hypothesis $\mathrm{HO}_{2 a}$, a significantly higher subjective perceived exertion, compared to the objectively determined exertion from the HR, was found during the "main part" of the VR-SET. With regard to the VR$\mathrm{ET}$, there were no differences between subjective perception and objective measurement. Accordingly, $\mathrm{HO}_{2 a}$ can be assumed for the VR-ET. Likewise, $\mathrm{HO}_{2 b}$ must be discarded due to the statistically significant difference between the two training sessions in the RPE $(n=22$, paired t-test $p=.028, \mathrm{Cl} 95 \%[1.54,24.82]$, $d=.502)$.

Table 4. Comparison of subjective perceived and objectively measured load. 


\begin{tabular}{|llllll|}
\hline & $\begin{array}{l}\text { Borg Scale } \\
\text { M (SD), bpm }\end{array}$ & HR & p-value & $95 \%$ Cl & d \\
\hline VR-SET $(\mathrm{n}=22)$ & $121.36(18.07)$ & $106.03(19.22)$ & $.010^{* *}$ & $-26.62,-4.05$ & -.602 \\
\hline VR-ET $(n=21)$ & $110.48(25.19)$ & $96.98(15.16)$ & .052 & $-27.15, .15$ & -.450 \\
\hline Paired t-test, $M$ mean, $S D$ standard deviation, $H R$ heart rate, $C l$ confidence interval, **p $<0.01$ \\
\hline
\end{tabular}

\section{Blood pressure}

The evaluation of BP was based on systolic and diastolic BP, as well as HR. Systolic baseline values were higher in the case of the VR-SET than the VR-ET before training ("pre") (Fig. 8). Over the course of the three post-exercise measurements ("post", " 5 min post" and "10 min post"), systolic BP decreased for both the VR-SET and the VR-ET (Fig. 8.). In the VR-SET, the tests of within-subjects effects revealed a significant main effect of training on systolic BP (ANOVA with repeated measures, Greenhouse-Geisser correction, $p<.001$, partial $\eta^{2}=.343, d=.722$ ). When systolic BP was measured before and after VR-ET, similar results were obtained (ANOVA with repeated measures, Sphericity assumed, $p<.001$, partial $\eta^{2}=$ $.551, d=1.108)$. The pairwise comparisons between the time points of each training session can be seen in Table 5. Accordingly, for systolic $\mathrm{BP}, \mathrm{HO}_{3}$ a can be rejected in relation to the VR-SET and the VR-ET. Moreover, 5 min after training, the systolic BP was significantly lower in the case of the VR-ET than the VR-SET (paired t-test $p=.020, \mathrm{Cl} 95 \%[-11.80,-2.51], \mathrm{d}=.535$ ). Regarding the time point " 5 min post", $\mathrm{HO}_{3 \mathrm{~b}}$ has to be rejected.

\section{Table 5. Pairwise comparisons of systolic BP in VR-SET and VR-ET.}




\begin{tabular}{|c|c|c|c|c|c|}
\hline (I) & $(J)$ & $M D(I-J)$ & SD & p-value & $95 \% \mathrm{Cl}$ \\
\hline \multicolumn{6}{|c|}{ VR-SET $(n=22)$} \\
\hline \multirow[t]{3}{*}{ pre } & post & .53 & 3.02 & 1.000 & $-8.25,9.31$ \\
\hline & $5 \mathrm{~min}$ post & 10.57 & 3.07 & $.015^{\star}$ & $1.62,19,51$ \\
\hline & 10 min post & 9.55 & 2.85 & $.018 *$ & $1.27,17,84$ \\
\hline \multirow[t]{2}{*}{ post } & $5 \mathrm{~min}$ post & 10.04 & 1.57 & $.000^{* * *}$ & $5.48,14,60$ \\
\hline & 10 min post & 9.02 & 1.89 & $.001^{\star \star}$ & $3.52,14,53$ \\
\hline $5 \mathrm{~min}$ post & 10 min post & -1.02 & 1.61 & 1.000 & $-5.69,3.66$ \\
\hline \multicolumn{6}{|c|}{ VR-ET $(n=22)$} \\
\hline \multirow[t]{3}{*}{ pre } & post & 3.21 & 1.98 & .718 & $-2.55,8.98$ \\
\hline & $5 \mathrm{~min}$ post & 9.75 & 2.35 & $.003^{\star \star}$ & $2.89,16.61$ \\
\hline & $10 \mathrm{~min}$ post & 16.07 & 2.34 & $.000 * \star \star$ & $9.24,22.89$ \\
\hline \multirow[t]{2}{*}{ post } & $5 \mathrm{~min}$ post & 6.54 & 1.52 & $.002^{\star \star}$ & $2.12,10.96$ \\
\hline & $10 \mathrm{~min}$ post & 12.86 & 1.87 & $.000^{* * *}$ & $7.43,18.29$ \\
\hline 5 min post & 10 min post & 6.32 & 1.74 & $.009 * \star$ & $1.26,11.38$ \\
\hline
\end{tabular}

For diastolic BP, a decrease in BP values after the training session was noticeable in both training programs (Fig. 8.). Although significant, within-subject effects were determined for the VR-SET (ANOVA with repeated measures, Sphericity assumed, $p<.046$, partial $\eta^{2}=.118, d=.366$ ) and VR-ET (ANOVA with repeated measures, Greenhouse-Geisser correction, $p=.002$, partial $\eta^{2}=.203, d=.505$ ), a significant difference between the time point "post" and "10 min post" was only detected for the VR-ET in the pairwise comparison (MD $=4.19 \pm 1.30, \mathrm{p}=.025, \mathrm{Cl} 95 \%[.40,7.98])$. Based on the significant main effects, $\mathrm{HO}_{3 \mathrm{a}}$ can be rejected for both training sessions. Moreover, no significant difference could be found between the two training programs at all four time points. Therefore, $\mathrm{HO}_{3 \mathrm{~b}}$ is retained for diastolic BP.

Whereas within the VR-ET the HR was, on average, almost constant between the time points (Fig. 8.) and thus no significant, within-subject effects could be recorded (ANOVA with repeated measures, Greenhouse-Geisser correction, $p=.079$, partial $\left.\eta^{2}=.101, d=.335\right)$, in the case of the VR-SET, internal significant main effects were found (ANOVA with repeated measures, Greenhouse-Geisser correction, $p<$ .001 , partial $\eta^{2}=.314, d=.677$ ). The pairwise comparison for the VR-SET showed differences between 
the time points "pre" and "post" (MD $=-6.80 \pm 1.65, \mathrm{p}=.003, \mathrm{Cl} 95 \%[-11.62,-1.99])$ as well as "post" and "10 min post" (MD = $3.93 \pm 1,24, \mathrm{p}=.028, \mathrm{Cl} 95 \%[.32,7,54])$. This leads to an acceptance of $\mathrm{HO}_{3 \mathrm{a}}$ in the case of the VR-ET and a rejection of the VR-SET. HR also differed between the VR-SET and the VR-ET. Accordingly, with a similar initial HR in both training sessions, a greater increase in HR was recorded for the VR-SET. This change resulted in significant differences between the training sessions at the time point "post" (paired t-test, $p=.004, \mathrm{Cl} 95 \%[3.34,10.66], \mathrm{d}=.693$ ), time point " 5 min post" (paired t-test, $\mathrm{p}=.001$, $\mathrm{Cl} 95 \%[2.60,8.69], \mathrm{d}=.821$ ) and time point "10 min post" (paired t-test, $\mathrm{p}=.002, \mathrm{Cl} 95 \%[2.15,8.27], \mathrm{d}=$ .752). Thus, $\mathrm{HO}_{3 b}$ was rejected.

Fig. 8. Progression of BP values at the respective measurement times.

\section{Perceived task load}

In terms of the perceived task load during VR training (Fig. 9), mental demands were significantly higher in the VR-ET than in the VR-SET $(n=22$, Wilcoxon test $p<0.001, r=-.800)$. In contrast, the VR-SET imposed significantly higher physical demands $(n=22$, Wilcoxon test $p=0.050, r=-.419)$. No differences were found with respect to temporal demands $(n=22$, Wilcoxon test $p=0.298, r=-.221)$. The effort expended to complete the tasks/exercises was significantly higher in the VR-SET than in the VR-ET $(n=$ 22 , Wilcoxon test $p=0.011, r=-.558$ ).

\section{Fig. 9. Boxplot of the adjusted task load values of the VR-SET and VR-ET.}

In addition, the majority of participants found both the total duration (14 participants in the VR-SET and 13 participants in the VR-ET) and the break times (17 participants in the VR-SET and 21 participants in the VR-ET) of the exergame appropriate. Eight participants in the VR-SET and nine participants in the VR$E T$, respectively, indicated that the total duration could be longer. In each training session, one person found that the breaks were too long. In the VR-SET, four people reported that the break times were too short.

\section{Discussion}

According to the title, two main aspects should be examined. The first purpose of the study was to investigate whether the intensity of the VR exergame was appropriate, so that older patients with hypertension could exercise within their individually calculated moderate load intensity of $40 \%$ to $60 \%$ during VR training.

In this regard, it was determined that a higher load intensity was achieved in the VR-SET than in the VRET. This behavior was also evident in the RPE, as the perceived exertion was significantly higher in the VRSET than in the VR-ET. In addition, significantly more participants reached the individual moderate exercise zone of 40 to $60 \%$ in the "main part" of the VR-SET. However, training within the individual load intensity of 40 to $60 \%$ of maximal HR in the "main part" of both training sessions was not significantly 
maintained over the time period. In relation to the main question, it can be seen that individuals with the developed VR exergame were limited able to train in their individual moderate load intensity. The reasons for this could be that the number of exercises, the number of repetitions and the number of sets performed are not yet sufficient to achieve moderate training loads. The speed of exercise execution and breaks, due to initially long exercise explanations, may also have led to a reduction in load intensity. Especially in the case of endurance training, the exercise explanations in the "main part" were significantly longer than for the strength endurance training, which might have led to different load intensities between the two training sessions. In addition, there were two to three participants who were unable to perform each exercise accurately, due to shoulder movement limitations, which may also have resulted in lower loading intensities. In other studies that collected HR measurements during the exergame with older participants, similar[35] or even lower[13] mean values were found, compared with the results of this study. Research by Vorwerg et al.[36] examined the load intensity of individuals in cardiac rehabilitation groups. It was found that around $65 \%$ of the participants trained within the individual training zone of 50 to $70 \%$.[36] The results illustrate that more individuals in conventional rehabilitation sports groups achieved individual load intensity than in this study. Gonçalves et al. on the other hand, found that participants spent more time participating in moderate to vigorous physical activity during an exergame than during a conventional workout.[37] For the development of the VR exergame, it can be stated that further adjustments (e.g., more exercises, more repetitions, more sets, less explanation time) must be implemented in the system. These adjustments should help increase the proportion of people who train within the individual load intensity.

The second aim was to evaluate whether VR exergames can have an impact on PB reduction among elderly patients with hypertension. In both training sessions, systolic BP was significantly reduced after training. Significant main effects were also recorded for diastolic BP. Despite the determined lower load intensity of the participants, a positive influence on BP could be found, which was characterized by a post-exercise hypotension for both VR training sessions. Although the load intensity in the VR-ET was significantly lower than in the VR-SET, higher mean differences were found for the VR-ET, especially for the time point 10 min post. Thus, the VR-ET displayed a stronger post-exercise hypotension. This clinically relevant phenomenon has already been studied numerous times and could also be found in conventional training methods for endurance or strength endurance.[38,39] However, this phenomenon is exclusively an acute BP reduction. Especially due to the lack of studies on VR and hypertension, the BP lowering effect must be further investigated by means of long-term studies for the target group.

With regard to the RPE, the present values almost corresponded to the conditions of Löllgen's recommendations or the ACSM exercise guidelines for adults with hypertension, which define moderate intensity as 11-13 on the Borg scale.[23,40] However, as highlighted by Ciolac et al. in their publication, it should be considered that an evaluation of the RPE after a single training session does not prove the statement that long-term training is perceived as equally strenuous.[41] In addition, it must be noted that the training program may have an influence on the RPE. Accordingly, in this study, lower RPE values were found for the VR-ET than for the VR-SET. These results are also reflected in the perceived task load, in which higher physical demands were recorded for the VR-SET. In particular, Morishita et al. maintain that 
in relation to strength training for the elderly, the Borg 6-20 RPE is a useful assessment for elderly individuals who perform resistance training.[42] However, training using the Borg scale can also lead to overload in patients with cardiovascular disease.[43] This could be a reason for the participants having a subjectively higher perceived exertion compared to the measured HR.

Another point is that according to the recommendations of cardiac societies, persons with hypertension should participate in endurance training five times a week for $30 \mathrm{~min}$.[8-10] As is also evident in this sample, older individuals are physically active on average two to three times per week. The gap between recommendations and the actual physical activity completed, was also described by Rhodes and de Bruijn in their publication.[44] To provide an additional incentive, VR exergames could help increase motivation for additional training sessions and, at the same time, achieve health-promoting effects. $[13,45]$

\section{Strength and limitations}

A strength of the study is that the comparability of HR and BP between the two training sessions is very high due to the identical study population.

However, there are also several limitations. Unfortunately, the calculated number of cases could not be retained, which may reduce the significance of the results. Furthermore, there are recommendations for the determination of post-exercise hypotension, which state that a measurement should be taken at least up to 20 min, or even better 120 min after training, in order to assess the effect.[38] The BP measurements in this study were only taken up to 10 min after training, which reduces the information regarding existing post-exercise hypotension. Due to the fact that the intervention only took place twice, it is not possible to draw any conclusions in relation to the long-term effect on BP.

Another point of concern relates to the individuals chosen to take part in the VR training sessions, which could have an impact on HR and BP. Conjectures suggest that older people might be afraid or excited due to the low acceptance of VR technologies. However, the results from other publications suggest that VR applications with regard to the health of older adults are not affected by negative attitudes.[46] In addition, our results show that BP was actually lower before the first VR training session than before the second training session. HR was also approximately the same before both VR training sessions. However, when we compare the groups of those who had VR experience and those who did not have VR experience, a difference in terms of HR became clear and may have had an impact.

A further significant limitation in the consideration of the load intensity and the BP relates to the medication. In the evaluation of the results, a more detailed consideration of the influence was omitted. However, as described, $\beta$-Blockers and angiotensin II receptor blockers, among others, lead to a reduced $\mathrm{HR}$ at rest as well as during exercise.[23] There was no instruction on standardized intake. 
Although the results of the Simulator Sickness Questionnaire (SSQ)[28] ruled out the presence of severe symptoms of VR sickness in this regard, the wearing of a VR headset during training may have led to an inhibition of movement action and thus to a lower load intensity. Due to the short intervention period of study dropouts with VR sickness symptoms, it was not possible to clarify how load intensity would relate to these symptoms. Other limitations that could have led to a lower load intensity would have been short signal interferences of the VR headset, which resulted in additional breaks. Even though the Polar M600 is considered suitable, erroneous measurements cannot be excluded. However, to preserve internal validity, the same watch was always used.

Future research needs to focus more on long-term intervention through VR exergames in patients with hypertension, so as to determine the actual effects on BP changes and load intensity. The methods and results presented in this study could be applied to future studies and provide a good basis for a randomized controlled trial.

\section{Conclusion}

Considering the importance of increasing hypertension prevalence in old age and the lower physical activity of older people, our results show that an exergame can contribute to positive health effects for older people with hypertension. Accordingly, post-exercise hypotension was found for both training sessions in our study. However, our results also showed that, on average, less than half of the participants trained within their individual moderate load intensities. In this context, continued adjustments to the VR exergame are essential. Nevertheless, VR exergames could be an alternative training method for seniors with hypertension to increase physical activity following the recommendations of cardiac associations. Moreover, the findings obtained can serve as a basis for further studies and have, therefore, focused for the first time on a target group of patients with hypertension. However, future studies need to examine the long-term benefits of VR exergame training for elderly patients with hypertension.

\section{Abbreviations}

ACC: American College of Cardiology, ACSM: American College of Sport Medicine, AHA: American Heart Association, BMI: body mass index, BP: blood pressure, CVD: cardiovascular diseases, Cl: confidence interval, ESC: European Society of Cardiology, ESH: European Society of Hypertension, HR: heart rate, HRR: heart rate reserve, ISH: International Society of Hypertension, max: maximum, M: mean, MD: mean difference, min: minimum, $\eta^{2}$ : Eta-Quadrat, RCTs: randomized controlled trials, RPE: Rating of Perceived Exertion, SD: standard deviation, SSQ: Simulator Sickness Questionnaire, TICS: Telephone Interview for Cognitive Status, VR: virtual reality, VR-SET: VR strength endurance training, VR-ET: VR endurance training.

\section{Declarations}




\section{Ethics approval and consent to participate}

All participants gave their written informed consent prior to participate in this study. The study was approved by the Ethics Committee of the Charité - Universitätsmedizin Berlin (EA1/019/20).

\section{Consent for publication}

Not applicable.

\section{Availability of data and materials}

The data set used and/or analyzed during the current study is available from the corresponding author upon reasonable request.

\section{Competing interests}

The authors declared that they have no competing interests.

\section{Funding}

The study was supported by the Federal Ministry of Education and Research Germany in the funding program: FPMTI2015-VR/AR: healthy living (Grant No. 16SV8066).

\section{Authors' contributions}

SV performed the main statistical analysis, interpreted the data and was a major contributor in writing the manuscript. OS contributed to project management, data collection and revision of manuscript. $\mathrm{MH}$ analyzed and interpreted the basic patient data, assisted in recruitment process, data collection and intervention protocols. All authors made editorial contributions to the manuscript. All authors read and approved the final manuscript.

\section{Acknowledgements}

The authors acknowledge the participants for their assistance during the study. We would also like to thank the Berlin University of Applied Sciences for providing the VITALAB.mobile.

\section{References}


1. Stanaway JD, Afshin A, Gakidou E, Lim SS, Abate D, Abate KH, et al. Global, regional, and national comparative risk assessment of 84 behavioural, environmental and occupational, and metabolic risks or clusters of risks for 195 countries and territories, 1990-2017: a systematic analysis for the global burden of disease study 2017. The Lancet. 2018,392:1923-94.

2. Neuhauser H, Kuhnert R, Born S. 12-Monats-Prävalenz von Bluthochdruck in Deutschland. Journal of Health Monitoring. 2017,2:57-63.

3. Benjamin EJ, Virani SS, Callaway CW, Chamberlain AM, Chang AR, Cheng S, et al. Heart disease and stroke statistics-2018 update: a report from the American Heart Association. Circulation. 2018, https://doi.org/10.1161/CIR.0000000000000558.

4. Mahabadi AA, Rassaf T. Bluthochdruck - Prävalenz, Bedeutung und Implikationen für die Prävention und Gesundheitsförderung. In: Tiemann M, Mohokum M, editors. Prävention und Gesundheitsförderung. Berlin, Heidelberg: Springer, 2021. p. 473-81.

5. Petrović I, Marinković M. Effects of different types of exercise programms on aterial blood pressure of the elderly. Facta Univ Ser Phys Educ Sport. 2019, 10.22190/FUPES181017064P.

6. Cornelissen VA, Smart NA. Exercise training for blood pressure: a systematic review and metaanalysis. JAHA. 2013, 10.1161/JAHA.112.004473.

7. Dickinson HO, Mason JM, Nicolson DJ, Campbell F, Beyer FR, Cook JV, et al. Lifestyle interventions to reduce raised blood pressure: a systematic review of randomized controlled trials. J Hypertens. 2006,24:215-33.

8. Whelton PK, Carey RM, Aronow WS, Casey DE, Collins KJ, Dennison Himmelfarb C, et al. 2017 ACC/AHA/AAPA/ABC/ACPM/AGS/APhA/ASH/ASPC/NMA/PCNA Guideline for the Prevention, Detection, Evaluation, and Management of High Blood Pressure in Adults. J Am Coll Cardiol. 2018,71:e127-248.

9. Unger T, Borghi C, Charchar F, Khan NA, Poulter NR, Prabhakaran D, et al. 2020 International Society of Hypertension global hypertension practice guidelines. Hypertension. 2020,75:1334-57.

10. Williams B, Mancia G, Spiering W, Agabiti Rosei E, Azizi M, Burnier M, et al. 2018 ESC/ESH Guidelines for the management of arterial hypertension: The Task Force for the management of arterial hypertension of the European Society of Cardiology (ESC) and the European Society of Hypertension (ESH). Eur Heart J. 2018,39:3021-104.

11. Robert Koch-Institut. Bericht Gesundheit in Deutschland 2015. Gesundheitsberichterstattung des Bundes Gemeinsam getragen von RKI und Destatista. 2015, 10.17886/rkipubl-2015-00.

12. Wollersheim D, Merkes M, Shields N, Liamputtong P, Wallis L, Reynolds F, et al. Physical and Psychosocial Effects of Wii Video Game Use among Older Women. Int J Emerg Technol Appl Eng Technol Sci. 2010,8:85-98.

13. Ogawa E, Huang H, Yu L-F, You T. Physiological responses and enjoyment of Kinect-based exergames in older adults at risk for falls: a feasibility study. Technol Health Care. 2019,27:353-62.

14. Lee LN, Kim MJ, Hwang WJ. Potential of Augmented Reality and Virtual Reality Technologies to Promote Wellbeing in Older Adults. Appl Sci. 2019, 10.3390/app9173556. 
15. McDonough DJ, Pope ZC, Zeng N, Liu W, Gao Z. Comparison of college students' blood pressure, perceived exertion, and psychosocial outcomes during Virtual Reality, exergaming, and traditional exercise: an exploratory study. Games Health J. 2020,9:290-6.

16. Huang $\mathrm{H}-\mathrm{C}$, Wong M-K, Lu J, Huang W-F, Teng C-I. Can using exergames improve physical fitness? A 12-week randomized controlled trial. Comput Hum Behav. 2017,70:310-6.

17. Silva LM da, Flôres FS, Matheus SC. Can exergames be used as an alternative to conventional exercises? Motriz: Rev Educ Fis. 2021, 10.1590/S1980-65742021019720.

18. Masoud M, Brinkmann C. Exergaming für PatientInnen mit metabolischem Syndrom?! Wien Med Wochenschr. 2020,170:171-7.

19. Hansen D, Stevens A, Eijnde BO, Dendale P. Endurance exercise intensity determination in the rehabilitation of coronary artery disease patients: a critical re-appraisal of current evidence. Sports Med. 2012,42:11-30.

20. Brandt J, Spencer M, Folstein M. The Telephone Interview for Cognitive Status. Cogn Behav Neurol. 1988,1:111-7.

21. Schmelter T, Rings S, Prasuhn C, Villwock J, Steinicke F, Hildebrand K. VITALab.Mobile - a mobile living lab. Proceedings of Mensch und Computer 2019. New York, NY, USA: Association for Computing Machinery, 2019. p. 917-920.

22. Tinetti ME, Williams TF, Mayewski R. Fall risk index for elderly patients based on number of chronic disabilities. Am J Med. 1986,80:429-34.

23. Liguori G, American College of Sports Medicine (ACSM). ACSMs Guidelines for Exercise Testing and Prescription. 11th ed. Philadelphia: Wolters Kluwer/Lippincott Williams \& Wilkins, 2021.

24. Borg G. Psychophysical scaling with applications in physical work and the perception of exertion. Scand J Work Environ Health. 1990,16:55-8.

25. Hart SG, Staveland LE. Development of NASA-TLX (Task Load Index): Results of Empirical and Theoretical Research. Advances in Psychology [Internet]. Elsevier, 1988 [cited 2020 Dec 15]. p. 13983. Available from: https://linkinghub.elsevier.com/retrieve/pii/S0166411508623869

26. Buchem I, Vorwerg S, Stamm O, Hildebrand K, Bialek Y. Gamification in Mixed-Reality exergames for older adult patients in a mobile immersive diagnostic center: a pilot study in the BewARe project. 2021 7th International Conference of the Immersive Learning Research Network (iLRN). IEEE, 2021. p. 1-8.

27. Buchem I, Vorwerg S, Stamm O, Kruschel K. Designing for rapport with virtual agents in a simulated Mixed Reality environment: results from a pilot study with senior users in the BewARe project. In: Ahram TZ, Falcão CS, editors. AHFE 2021: Advances in Usability, User Experience, Wearable and Assistive Technology. Springer International Publishing, 2021. p. 140-8.

28. Stamm 0, Vorwerg S. Virtual Reality sickness evaluation in exergames for older hypertensive patients: a comparative study of training methods in a virtual environment. In: Chen JYC, Fragomeni G, editors. Virtual, Augmented and Mixed Reality. Cham: Springer International Publishing, 2021. p. $134-46$. 
29. Polar. What is optical heart rate tracking? [Internet]. Polar Blog. 2017 [cited 2021 Aug 31]. Available from: https://www.polar.com/blog/optical-heart-rate-tracking-polar/

30. Zhang Y, Weaver RG, Armstrong B, Burkart S, Zhang S, Beets MW. Validity of Wrist-Worn photoplethysmography devices to measure heart rate: A systematic review and meta-analysis. $J$ Sports Sci. 2020,38:2021-34.

31. Horton JF, Stergiou P, Fung TS, Katz L. Comparison of Polar M600 optical heart rate and ECG heart rate during exercise. Med Sci Sports Exerc. 2017,49:2600-7.

32. Edwards S. The Heart Rate Monitor Book. 5th ed. Pennsylvania State University: Polar CIC, 1993.

33. Karvonen MJ. The effects of training on heart rate: A longitudinal study. Ann Med Exp biol Fenn. 1957,35:307-15.

34. Cohen J. Statistical Power Analysis for the Behavioral Sciences. 2nd ed. New York: Routledge, 1988.

35. Alves da Cruz MM, Ricci-Vitor AL, Bonini Borges GL, Fernanda da Silva P, Ribeiro F, Marques Vanderlei LC. Acute hemodynamic effects of Virtual Reality-based therapy in patients of cardiovascular rehabilitation: a cluster randomized crossover trial. Arch Phys Med Rehabil. 2020,101:642-9.

36. Vorwerg S, Stamm O, Menant A, Alex S, Müller-Werdan U. Observational study in cardiac rehabilitation groups phase III: a comparison of perceived and measured training intensity during a moderate-intensity workout. Eur J Phys Rehabil Med. 2021,57:414-23.

37. Gonçalves AR, Muñoz JE, Gouveia ÉR, Cameirão M da S, Bermúdez i Badia S. Effects of prolonged multidimensional fitness training with exergames on the physical exertion levels of older adults. Vis Comput. 2021,37:19-30.

38. Brito LC de, Fecchio RY, Peçanha T, Lima A, Halliwill J, Forjaz CL de M. Recommendations in postexercise hypotension: concerns, best practices and Interpretation. Int J Sports Med. 2019,40:487-97.

39. Wegmann M, Hecksteden A, Poppendieck W, Steffen A, Kraushaar J, Morsch A, et al. Postexercise hypotension as a predictor for long-term training-induced blood pressure reduction: a large-scale randomized controlled trial. CJSM. 2018,28:509-515.

40. Löllgen H. Bedeutung und Evidenz der körperlichen Aktivität zur Prävention und Therapie von Erkrankungen. Dtsch Med Wochenschr. 2013,138:2253-9.

41. Ciolac E, Mantuani S, Neiva C, Verardi C, Pessôa-Filho D, Pimenta L. Rating of perceived exertion as a tool for prescribing and self regulating interval training: a pilot study. Biol Sport. 2015,32:103-8.

42. Morishita S, Tsubaki A, Nakamura M, Nashimoto S, Fu JB, Onishi H. Rating of perceived exertion on resistance training in elderly subjects. Expert Rev Cardiovasc Ther. 2019,17:135-42.

43. Yasumura K, Abe H, Kato T, Idemoto A, Shinouchi K, Mishima T, et al. Predictive Factors for Overloaded Rehabilitation Using Borg Scale in Patients with Heart Failure. J Card Fail. 2016,22:S185.

44. Rhodes RE, de Bruijn G-J. How big is the physical activity intention-behaviour gap? A meta-analysis using the action control framework. Br J Health Psychol. 2013,18:296-309.

45. Costa MTS, Vieira LP, Barbosa E de O, Mendes Oliveira L, Maillot P, Otero Vaghetti CA, et al. Virtual Reality-based exercise with exergames as medicine in different contexts: a short review. Clin Pract 
Epidemiol Ment Health. 2019, 10.2174/1745017901915010015.

46. Huygelier H, Schraepen B, van Ee R, Vanden Abeele V, Gillebert CR. Acceptance of immersive headmounted virtual reality in older adults. Sci Rep. 2019, 10.1038/s41598-019-41200-6.

\section{Figures}

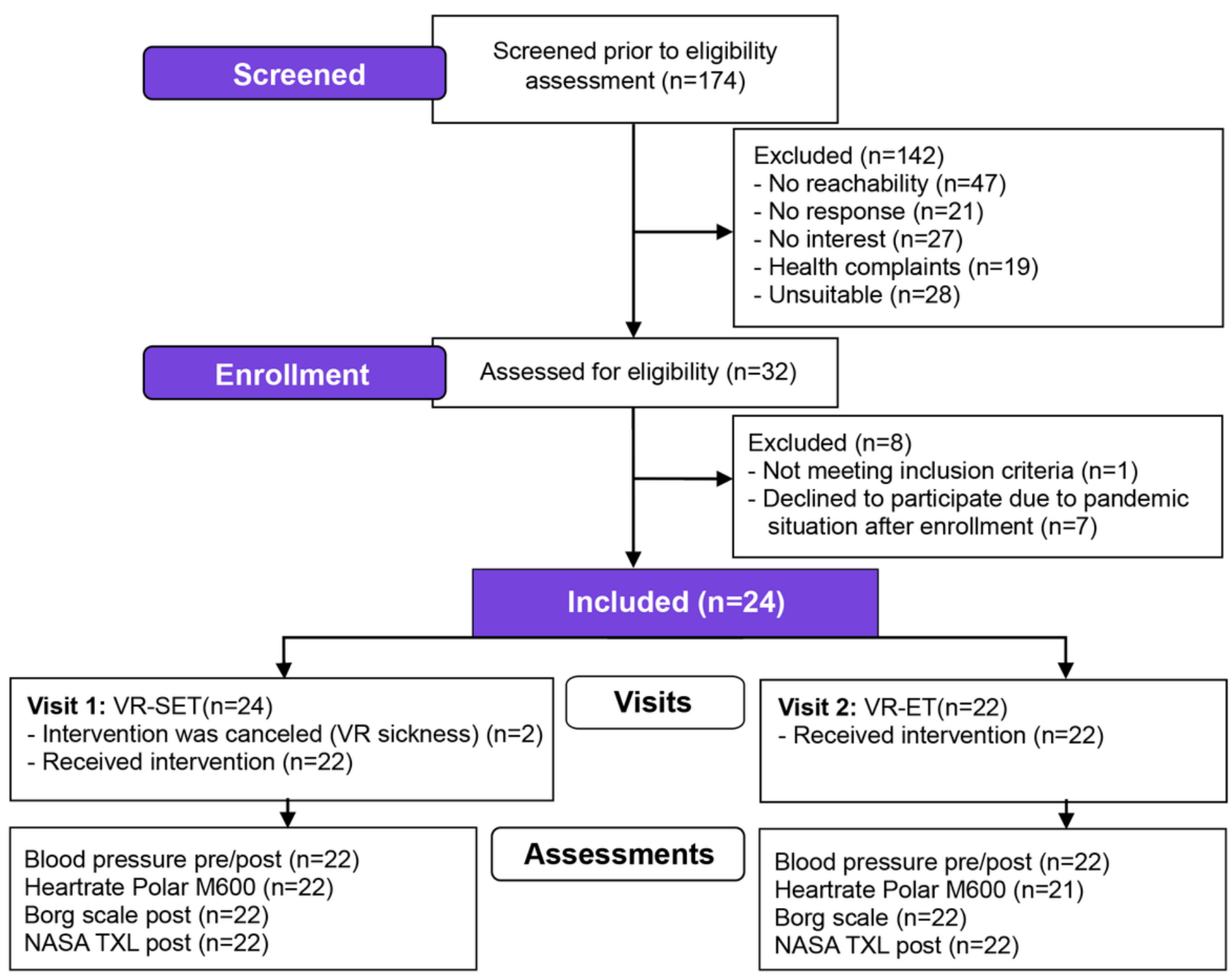

\section{Figure 1}

Flow diagram of the recruitment process, visits and assessments 


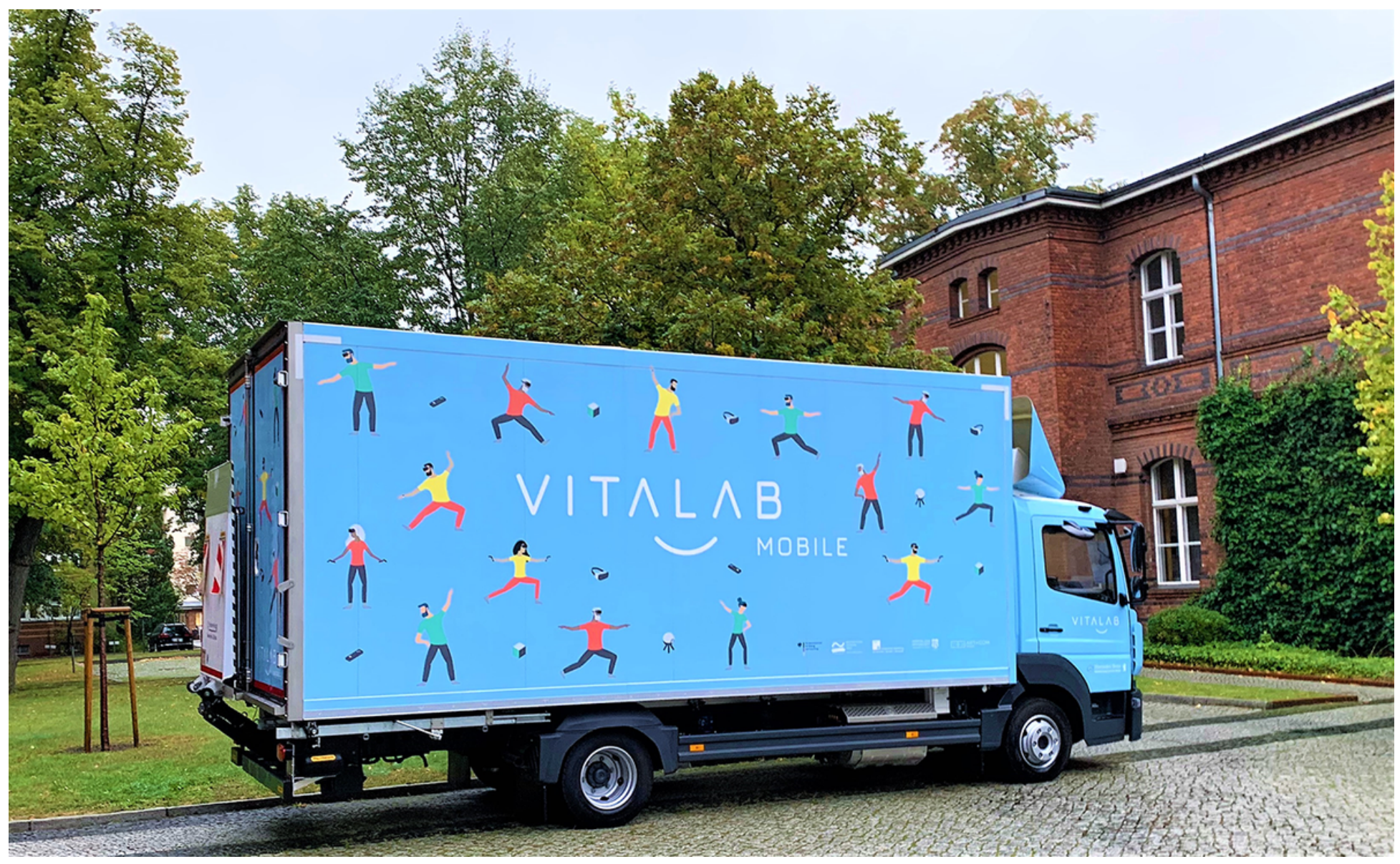

Figure 2

Mobile research laboratory VITALab.mobile. 
Section 1. introduction 2 min

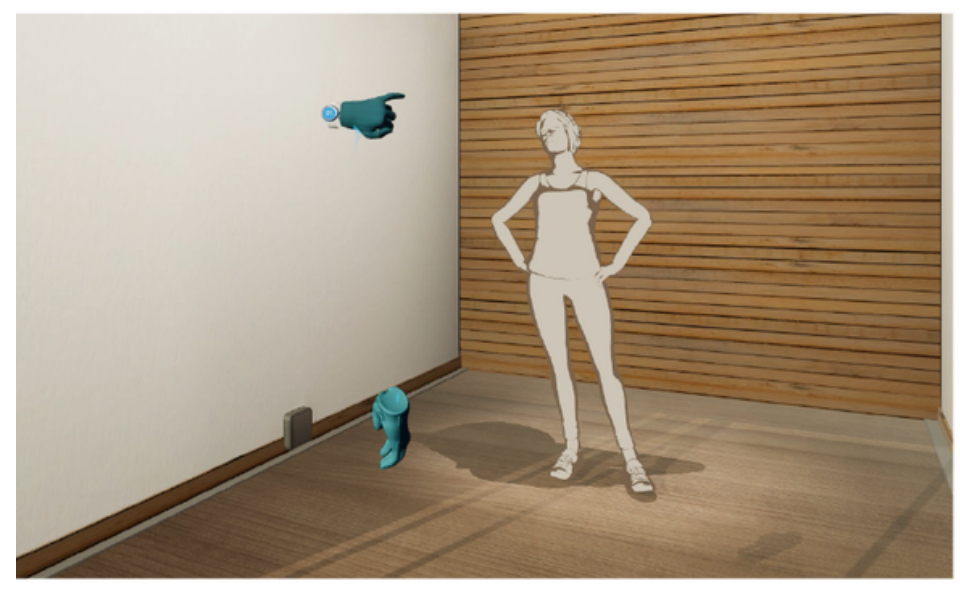

Section 4. cool down $1 \mathrm{~min}$

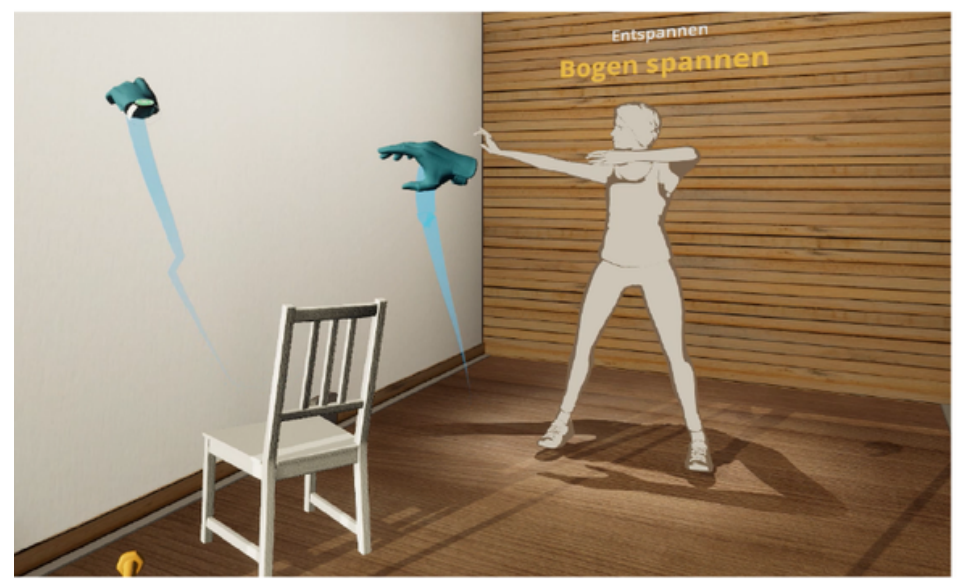

Section 2. warm up $1 \mathrm{~min}$

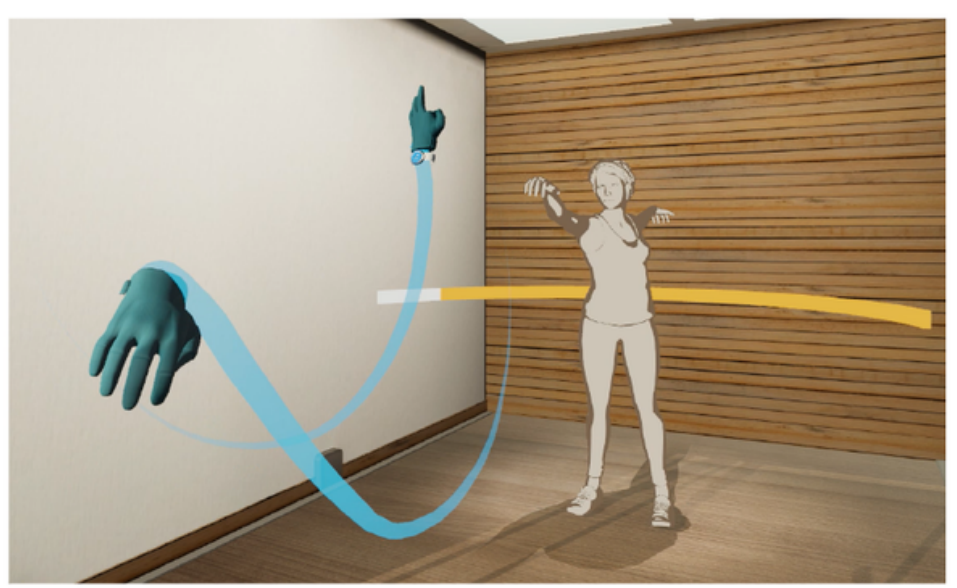

Section 5. training analysis $1 \mathrm{~min}$

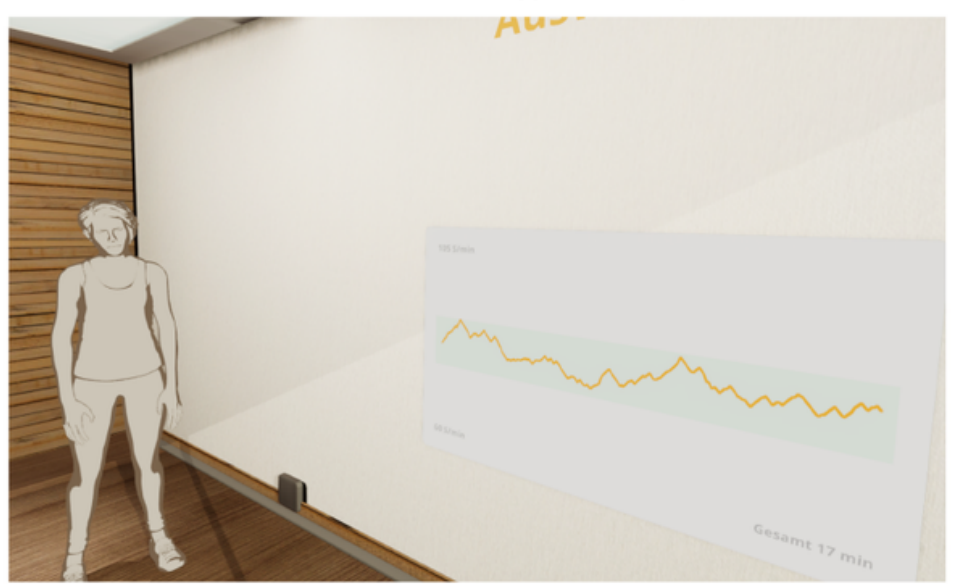

Figure 3

Section 1, 2, 4 and 5 of the VR Exergame 
Exercise 1. Squat 20 repetitions

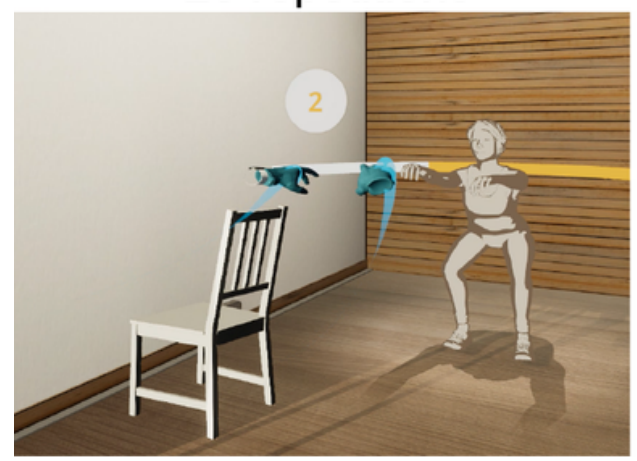

Exercise 4. Leg raise 12 repetitions

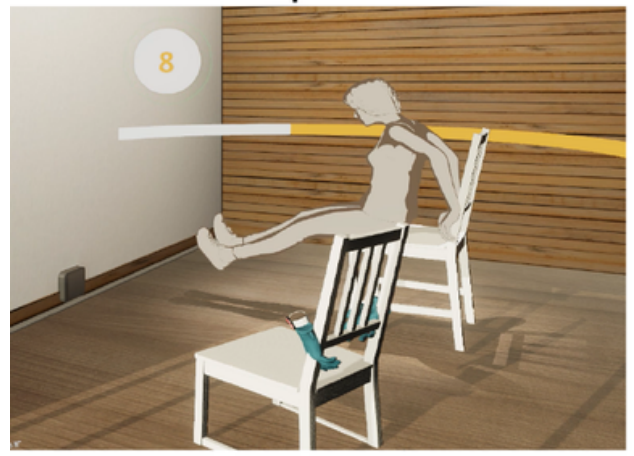

Exercise 2. Overhead press 20 repetitions

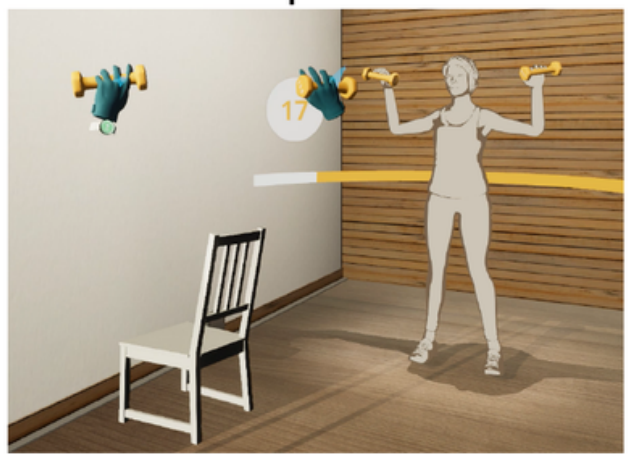

Exercise 5. Heel raise 20 repetitions

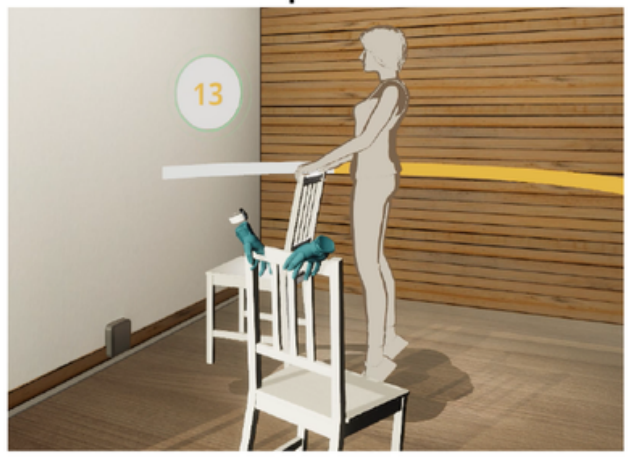

Exercise 3. Lateral raise 20 repetitions

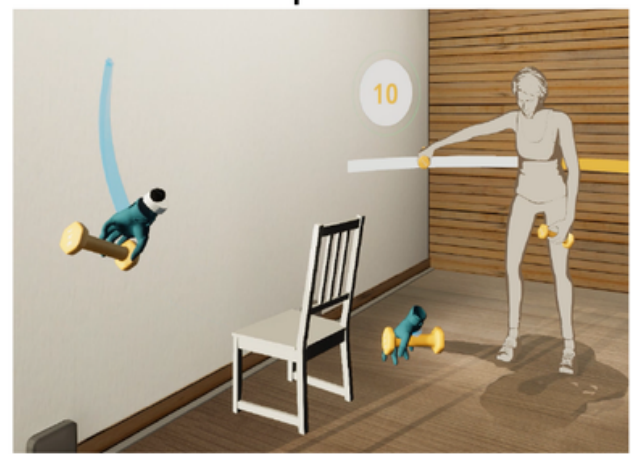

Active break 1 Minute

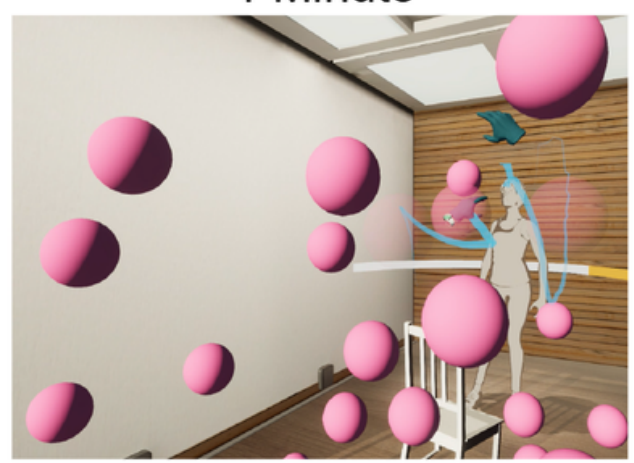

Figure 4

Section 3 - training phase of the VR-SET (visit 1).

Exercise 1. Ball Game

2-3 Minutes

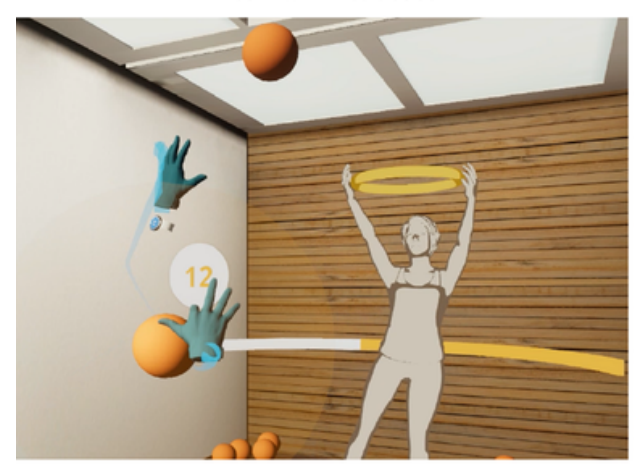

Exercise 2. High five 2-3 Minutes

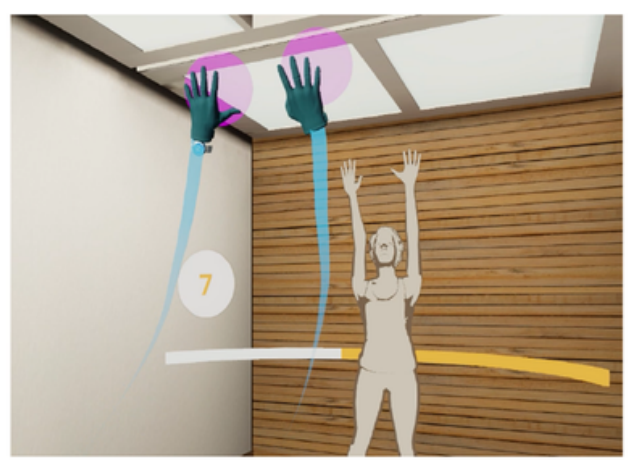

Exercise 3. Hustle dance 15 Minutes

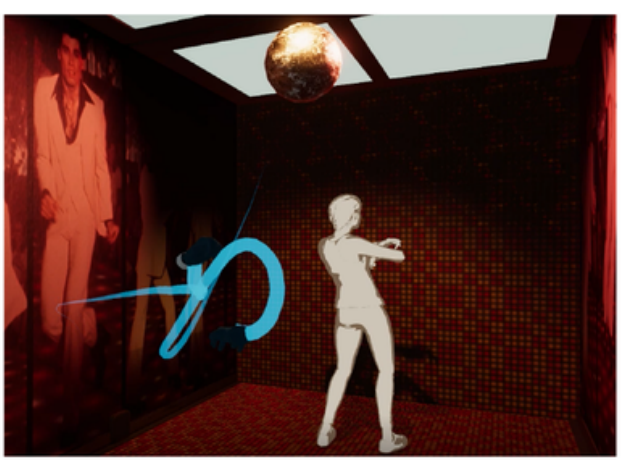

Figure 5

Section 3 - training phase of the VR-ET (visit 2). 

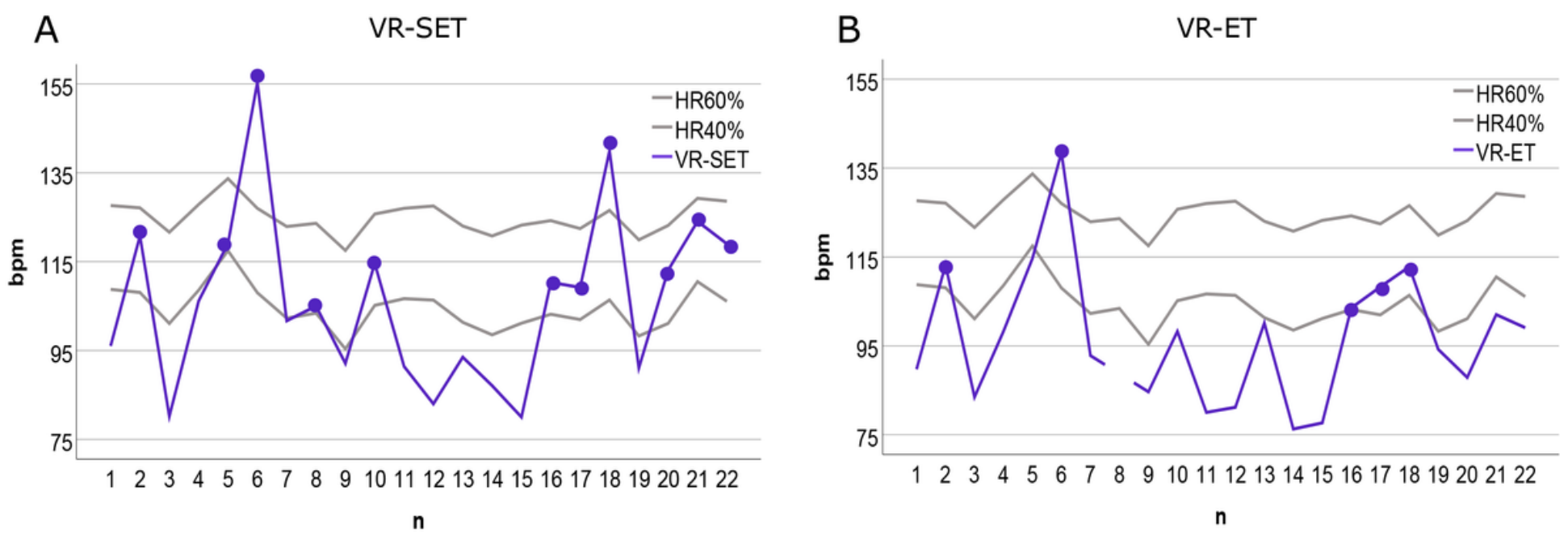

Figure 6

Progression of mean HR during training phase in VR-SET and VR-ET per participant. The black circles indicate exceeding an individual load intensity of 40 \%. Participant 8 in Fig. B is a missing value. 


\section{VR-SET}

A

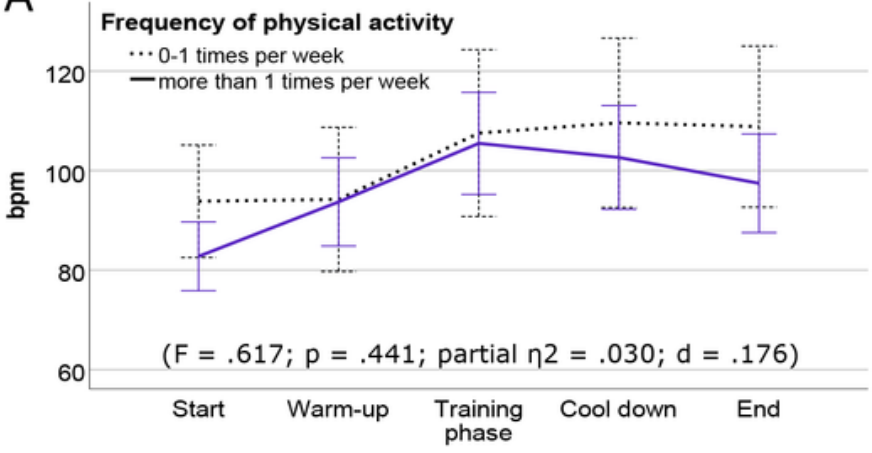

B

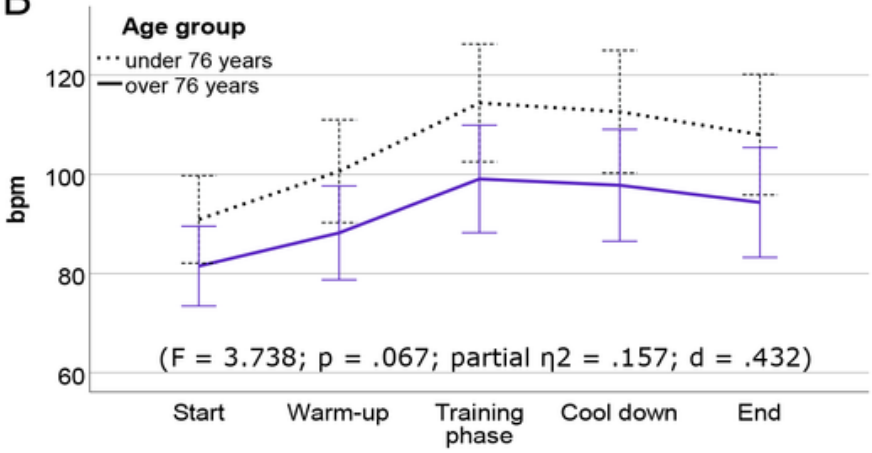

C
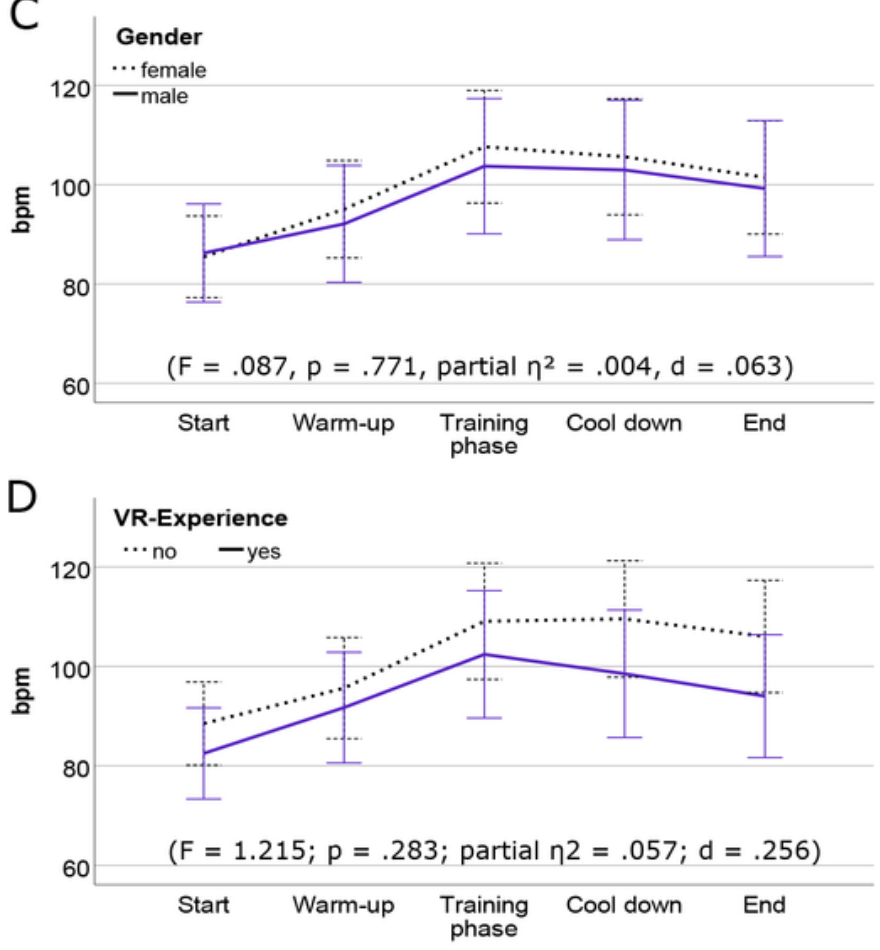

VR-ET

$E$

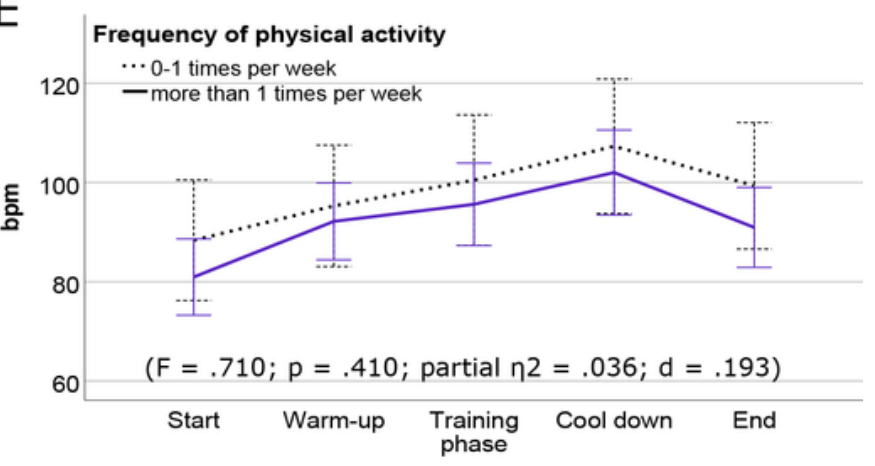

$\mathrm{F}$

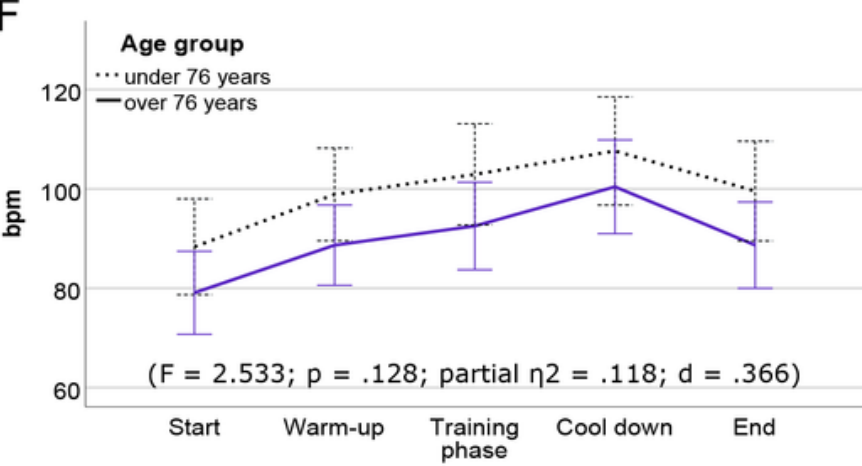

G
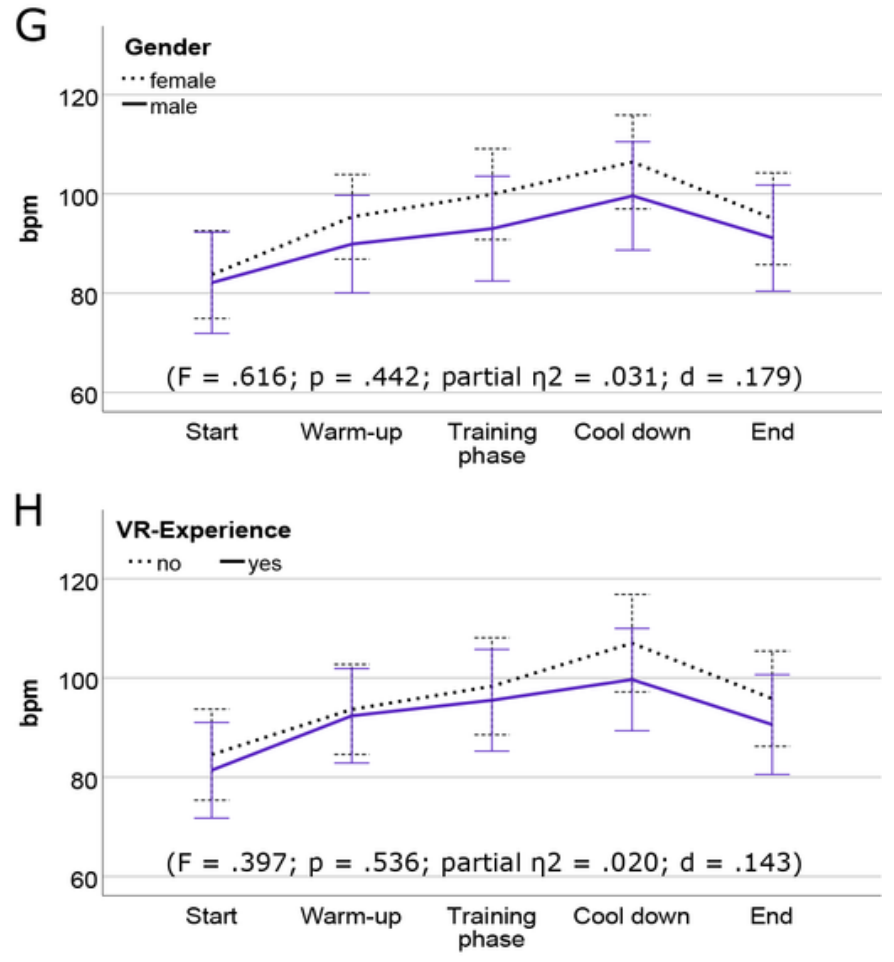

\section{Figure 7}

Main Effects of ANOVA with repeated measures for groups in VR-SET and VR-ET. 

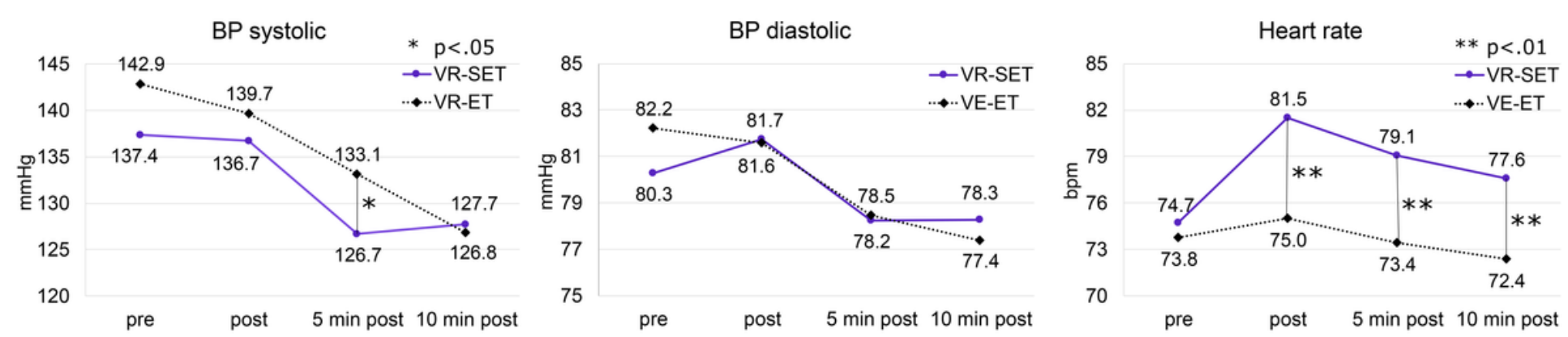

Figure 8

Progression of BP values at the respective measurement times.

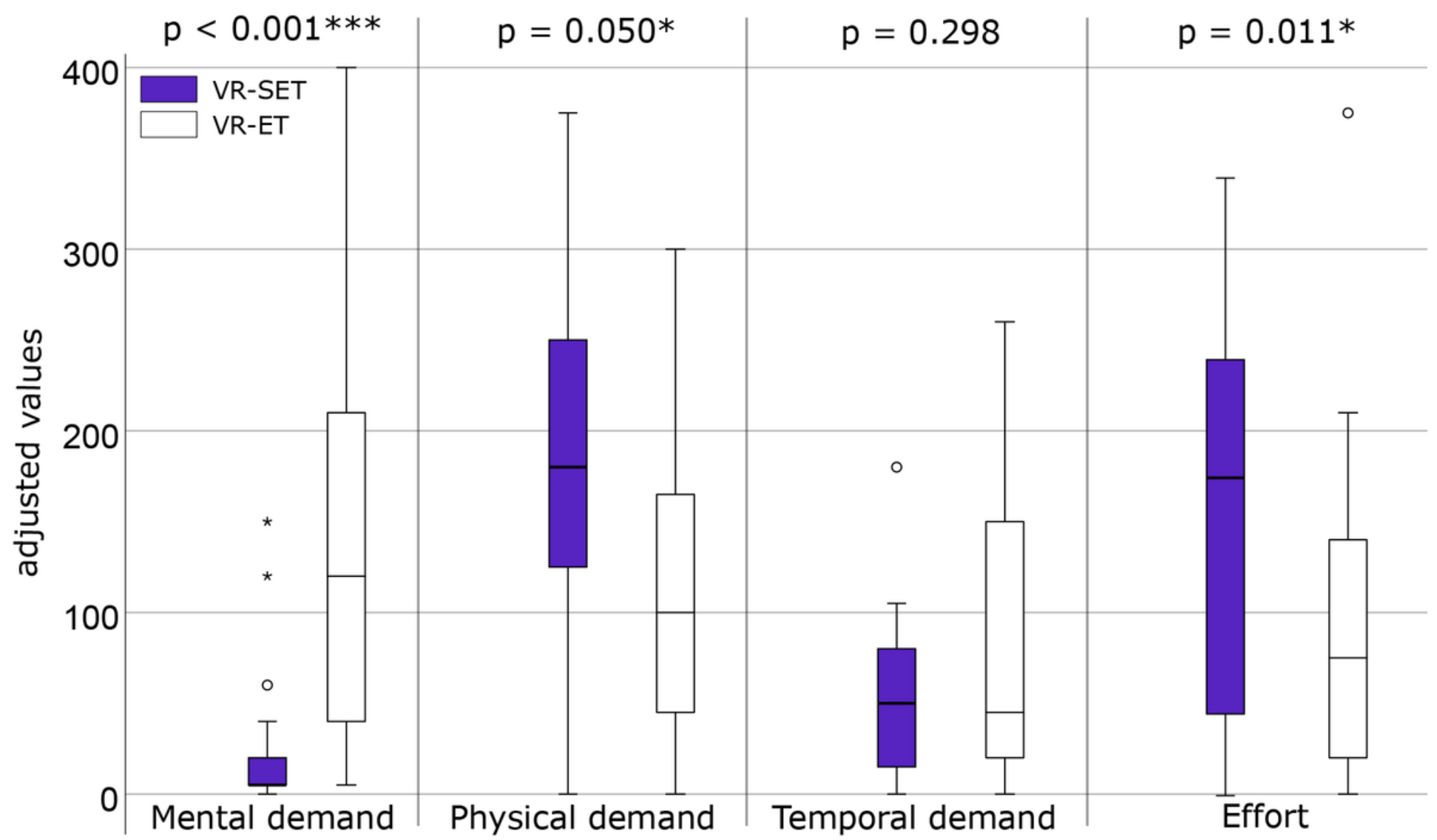

Figure 9

Boxplot of the adjusted task load values of the VR-SET and VR-ET. 